\title{
Mechanical Injury of the Subthalamic Area During Stereotactic Surgery Followed by Improvement of Trunk, Neck, and Face Tremor -Case Report-
}

\author{
Yutaka HiRASHIMA, Hiroaki IKEDA, Takashi ASAHI, Takashi SHIBATA, \\ Kyo NoguCHI*, Fumio SHIMA**, and Shunro ENDO
}

\begin{abstract}
Departments of Neurosurgery and * Radiology, Toyama Medical and Pharmaceutical University, Toyama; ${ }^{* *}$ Department of Neurosurgery, Kaizuka Hospital, Fukuoka
\end{abstract}

\begin{abstract}
A 50-year-old man had undergone right nucleus ventrointermedius (Vim) thalamotomy 1 year previously, resulting in the disappearance of left hand tremor. However, he presented with right distal and proximal tremor including the axial trunk, neck, and head. Deep brain stimulation (DBS) of the left Vim for these symptoms was unsuccessful. Attempts were made to stimulate the left Vim, nucleus ventralis lateralis, and subthalamic nucleus (STN), but no significant improvement was obtained after repeat surgery. However, subsequent improvement of the symptoms including proximal tremor was very marked even without DBS stimulation. Brain magnetic resonance imaging demonstrated lesion and edema in the posteromedial area of the STN. Mechanical injury of the area caused by the surgical procedures may have contributed to the improvement in his persistent symptoms.
\end{abstract}

Key words: essential tremor, proximal tremor, posteromedial subthalamic area

\section{Introduction}

Stereotactic thalamotomy such as nucleus ventrointermedius (Vim) thalamotomy is an established treatment for distal tremor in patients with Parkinson's disease and essential tremor. However, tremor of the axial trunk, neck, and head is very difficult to treat by lesioning or deep brain stimulation (DBS) of the thalamus. ${ }^{3)}$ We treated a patient whose persistent tremor improved unexpectedly after multiple stereotactic surgeries with various targets and discuss the possible mechanism for the improvement of symptoms.

\section{Case Report}

A 50-year-old man originally presented with left upper extremity action tremor in 1983. Although consumption of alcohol reduced his symptoms, medication provided by two clinics was not effective. A diagnosis of essential tremor was established at the Department of Neurology of a local hospital in

Received July 1, 2004; Accepted March 18, 2005
1995. Arotinol hydrochloride and clonazepam were partially effective. However, his symptoms persisted. He consented to be transferred to the Department of Neurosurgery, Toyama Medical and Pharmaceutical University for stereotactic surgery. He underwent right Vim thalamotomy on October 2, 1997 and his symptoms disappeared completely immediately after surgery.

Tremor appeared again in the opposite upper extremity, neck, head, and trunk, and he needed a cane to maintain his balance from the beginning of 1998. DBS of the left Vim was planned because the right Vim had already been lesioned, and was performed on a target located 13-15 mm lateral and 2-3 $\mathrm{mm}$ posterior to the mid-commissural point at the level of the anterior commissure-posterior commissure line on August 18, 1998 and March 27, 1999. However, DBS resulted in no improvement of symptoms during surgery and sometimes caused dysesthesia. At the patient's request, he was transferred to the Department of Neurosurgery, Kaizuka Hospital. The left Vim, nucleus ventralis lateralis (VL), and subthalamic nucleus (STN) were stimulated during a prolonged surgical procedure on April 


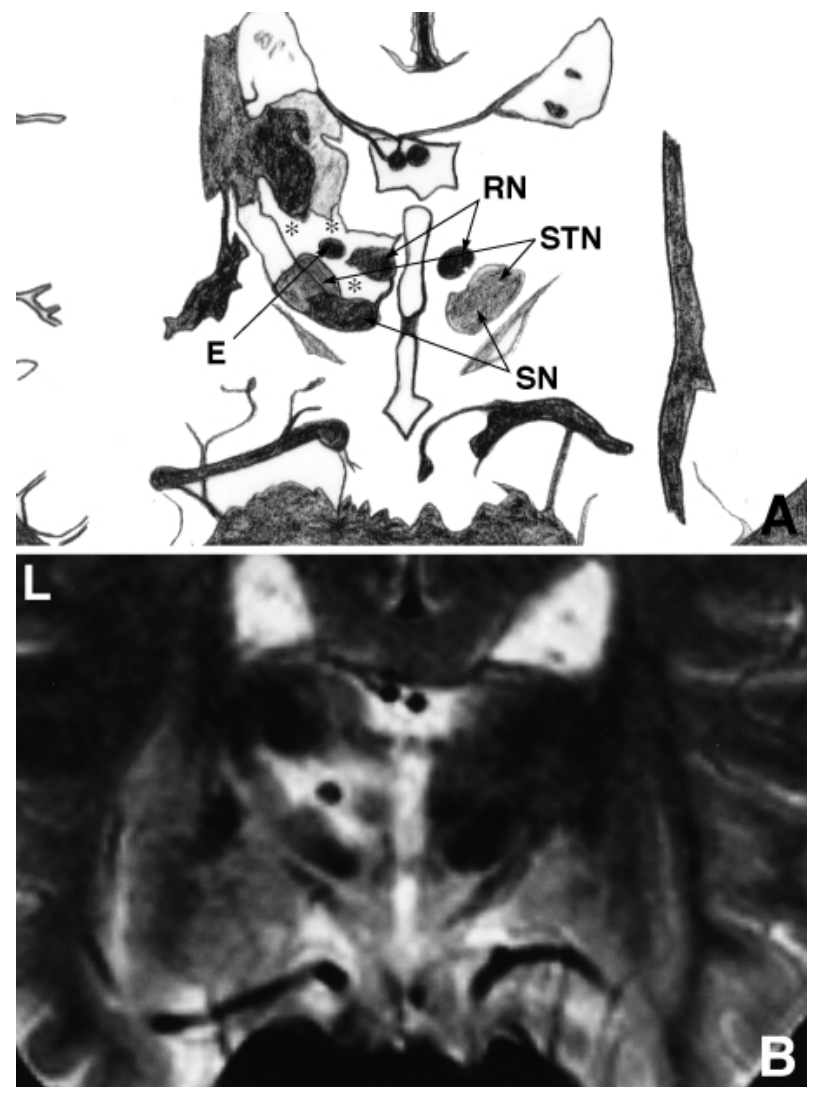

Fig. 1 A: Schema illustrating the features of $T_{2^{-}}$ weighted magnetic resonance (MR) imaging (B). B: $T_{2}$-weighted MR image taken immediately after surgery for stimulation of the nucleus ventrointermedius, nucleus ventralis lateralis, and subthalamic nucleus (STN) showing migration of the electrode (E), and injury and edema in the white matter of the posteromedial subthalamic area (asterisks). $\mathrm{RN}$ : red nucleus, $\mathrm{SN}$ : substantia nigra.

23, 2000 and electrodes were temporarily implanted in the VL. Magnetic resonance (MR) imaging subsequently demonstrated migration of the electrodes, which were reimplanted in the left Vim-nucleus ventro-oralis posterior on April 30, 2000 (Fig. 1). MR imaging also showed injury and edema in the white matter of the posteromedial subthalamic area.

The tremor of the right upper and lower extremities subsided, but an increase in DBS amplitude induced dysarthria and numbness of the right side of the face. We continued to follow up the patient after discharge from Kaizuka Hospital. His tremor disappeared rapidly and his gait improved to the point that he could walk without a cane 3 months after the final surgery. He switched off the DBS system due to its side effects, and found that

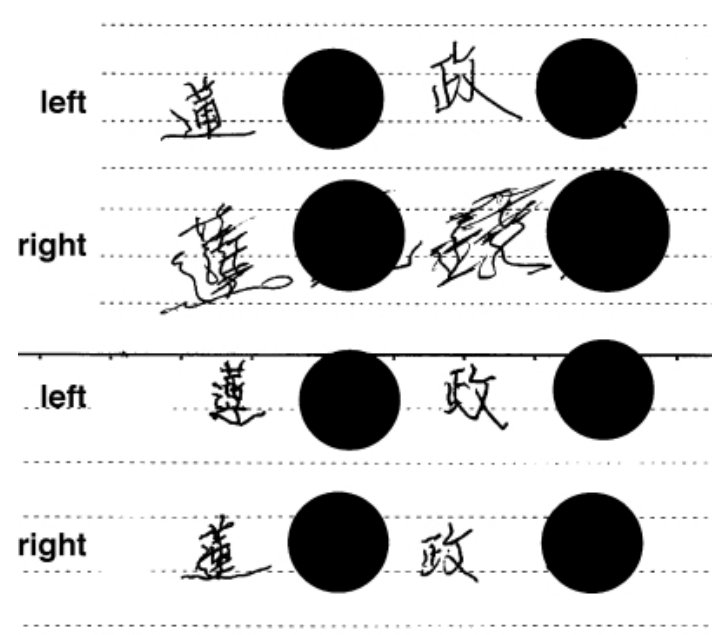

Fig. 2 Comparison of the patient's writing before final surgery (upper, 1999.3.30) and 11 months after final surgery (lower, 2004.3.16).

improvement of the symptoms was retained without DBS stimulation. Recently he volunteered his services and found employment. He does not want to remove the DBS system or undergo MR imaging because he is fearful of worsening symptoms.

\section{Discussion}

The mechanism of tremor generation is thought to involve fibers running from the cerebellar nuclei and the mesencephalic reticular formation to the thalamus. ${ }^{2,4,6)}$ Afferent fibers run to the zona incerta (ZI). These fibers are more compactly bundled in the subthalamus than the thalamus. Conversely, the fibers and neurons are more widely distributed in the Vim or VL than in the subthalamus. $\left.{ }^{7}\right)$ Widespread symptoms are difficult to control using a similar lesion or electrode in the thalamus to that used in the subthalamus. Therefore, intervention in the posteromedial subthalamic area including the ZI may improve proximal tremor that is refractory to Vim thalamotomy. ${ }^{5}$ Thalamic surgery in patients with multiple sclerosis had obtained tremor suppression by lower stimulation voltages within $13 \mathrm{~mm}$ of the thalamic target along the trajectory. ${ }^{1)}$ This area is located ventral to the VL, posteromedial to the STN, and lateral to the red nucleus.

In the present patient, multiple stereotactic surgeries with targets in the Vim, VL, and STN were performed for tremor of the right upper extremity, trunk, face, and neck. Although we could not obtain new information about the subthalamic area from MR imaging, the lesions and perilesional edema had 
been observed on brain MR imaging before the final surgery. The lesions and edema in the posteromedial subthalamic area appeared to be more severe after the final surgery.

Our patient showed gradual improvement and long-term stabilization of symptoms after surgery. This course is somewhat different to the typical course of symptoms after stereotactic surgery for involuntary movement targeting only the thalamus or globus pallidus. The improvement of symptoms was also maintained after surgery at the posteromedial subthalamic area in patients with multiple sclerosis. ${ }^{1)}$ Surgery in this area was effective in improving writing. ${ }^{5)}$ In our patient, writing was also observed to improve markedly after the final surgery (Fig. 2). The favorable result in the present case may be attributed to reciprocal actions between the interventions in the thalamus and STN, but the outcome suggests that stereotactic surgery including lesioning and DBS of the posteromedial subthalamic area may be a potential treatment for tremor in the axial trunk or multiple extremities.

\section{References}

1) Alusi SH, Aziz TZ, Glickman S, Jahanshahi M, Stein JF, Bain PG: Stereotactic lesional surgery for the treatment of tremor in multiple sclerosis: a prospec- tive case-controlled study. Brain 124: 1576-1589, 2001

2) Deuschl G, Bain P: Pathophysiology of nonparkinsonian tremors. Mov Disord 17(Suppl 3): S41-S48, 2002

3) Deuschl G, Bain P: Deep brain stimulation for tremor: Patient selection and evaluation. Mov Disord 17(Suppl 3): S102-S111, 2002

4) Mitrofanis J, de Fonseka R: Organisation of connections between the zona incerta and the interposed nucleus. Anat Embryol (Berl) 204: 153-159, 2001

5) Murata J, Kitagawa M, Uesugi H, Saito H, Iwasaki Y, Kikuchi S, Tashiro K, Sawamura Y: Electrical stimulation of the posterior subthalamic area for the treatment of intractable proximal tremor. J Neurosurg 99: 708-715, 2003

6) Velasco FC, Molina-Negro P, Bertrand C, Hardy J: Further definition of the subthalamic target for arrest of tremor. J Neurosurg 36: 184-191, 1972

7) Vitek JL, Ashe J, De Long MR, Alexander GE: Physiologic properties and somatotopic organization of the primate motor thalamus. J Neurophysiol 71: 1498-1513, 1994

Address reprint requests to: Y. Hirashima, M.D., Department of Neurosurgery, Toyama Medical and Pharmaceutical University, 2630 Sugitani, Toyama, Toyama 930-0194, Japan.

e-mail: yhira@ms.toyama-mpu.ac.jp 